\title{
Fluorescence Microscopy of Single Liposomes with Incorporated Pigment-Proteins
}

Marijonas Tutkus ${ }^{\ddagger}$, Parveen Akhtar ${ }^{\S}$, Jevgenij Chmeliov ${ }^{\dagger}$, Fanni Görföl ${ }^{\S}$. Gediminas Trinkunas ${ }^{\ddagger}$, Petar H. Lambrev ${ }^{\S}$, Leonas Valkunas $\dagger^{*}$.

†Department of Molecular Compound Physics, Centre for Physical Sciences and Technology, Saulètekio Avenue 3, LT-10257 Vilnius, Lithuania

$\dagger$ Institute of Chemical Physics, Faculty of Physics, Vilnius University, Vilnius, Lithuania, Sauletekio Avenue 9-III, LT-10222 Vilnius, Lithuania

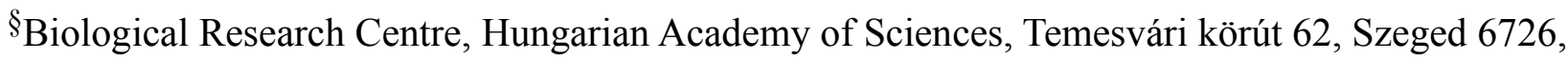
Hungary

*e-mail: leonas.valkunas@ff.vu.lt. 


\begin{abstract}
Reconstitution of transmembrane proteins into liposomes is a widely used method to study their behavior under conditions closely resembling the natural ones. However, this approach does not allow precise control of the liposome size, reconstitution efficiency and the actual protein-to-lipid ratio in the formed proteoliposomes, which might be critical for some applications and/or interpretation of data acquired during the spectroscopic measurements. Here we present a novel strategy employing methods of proteoliposome preparation, fluorescent labelling, purification, and surface immobilization that allow us to quantify these properties using fluorescence microscopy at the single-liposome level and for the first time apply it to study photosynthetic pigment-protein complexes LHCII. We show that LHCII proteoliposome samples, even after purification with a density gradient, always contain a fraction of non-reconstituted protein and are extremely heterogeneous in both protein density and liposome sizes. This strategy enables quantitative analysis of the reconstitution efficiency of different protocols and precise fluorescence spectroscopic study of various transmembrane proteins in a controlled native-like environment.
\end{abstract}




\section{Introduction}

Photosynthesis is the natural process utilized by plants, algae, and some bacteria to collect solar energy and store it in the form of chemical bonds. In green plants, the light reactions of photosynthesis occur in the pigment-protein supercomplexes embedded into the thylakoid membranes of the chloroplast. The process starts with the absorption of a photon of sunlight by a pigment molecule bound to the lightharvesting proteins, followed by the transfer of generated electronic excitation energy to the reaction center (RC). There the excitation energy initiates an electron transfer process to build up an electrochemical potential across the thylakoid membrane that fuels chemical reactions producing energy-rich complex carbohydrates ${ }^{1}$. Besides this primary function, multiple level self-regulatory processes occur in the thylakoid membrane aiming to optimize the photosynthesis efficiency under varying light conditions. This optimization involves continuous rebuild of the pigment-protein complexes, diffusion of the antenna complexes through the membrane during the state transition, dynamic variation of the size of the light-harvesting antenna attached to the RC, or dissipation of the excess excitation energy via non-photochemical quenching (NPQ) that is reversibly activated during strong sunlight $t^{2-4}$.

Due to the presence of two types of photosystems within the membrane as well as two types of excitation quenchers (RCs and NPQ-traps), the spectroscopic signatures of various parallel processes occurring in the thylakoid membrane are not easily resolved ${ }^{5-9}$, which severely complicates the detailed direct investigation of the molecular mechanisms involved. Instead, different photosynthetic units or just pigment-protein complexes are usually extracted from the thylakoid membrane and then are studied separately either in the detergent-solubilized form utilizing conventional bulk spectroscopy methods ${ }^{10-17}$ or being immobilized on some surface while applying the single-molecule microscopy techniques ${ }^{18-22}$. Such treatment in the non-native environment allows much more straightforward analysis of the collected data but does not ensure that all the observations directly correspond to the in 
vivo processes and not to the side effect of the detergent micelle environment. This drawback, however, may be overcome by studying isolated pigment-protein complexes embedded into liposomesartificial lipid bilayers that mimic natural thylakoid membranes ${ }^{14,23-26}$. Liposomes are useful model systems for reconstitution of the transmembrane and membrane-related proteins, which allow studying their properties in a native-like environment bypassing their complex cellular surrounding. Reconstitution of proteoliposomes permits a high level of flexibility achieved by varying lipid and protein composition as well as lipid-to-protein $(\mathrm{L}: \mathrm{P})$ ratio ${ }^{14,15}$. On the other hand, reproduction of proteoliposomes with fixed L:P ratio, independent of the liposome size, remains a great challenge en $^{23,27-}$ ${ }^{29}$. Therefore, the variability of the mentioned parameters in the studied sample might play a significant role in proteoliposome's fluorescence quenching, sensitive to protein lateral aggregation, conformational switches, and protein-lipid interactions, possibly skewing the ensemble-type measurement results or even leading to their miss-interpretation.

Recent studies of the proteoliposomes reconstituted with the major light-harvesting complex of photosystem II from plants (LHCII) revealed several valuable insights. First of all, quantum yield (QY) of LHCII fluorescence in liposomes is generally lower than of the ones solubilized in detergent micelles $^{30}$. Secondly, QY could be varied by changing the L:P ratio affecting the number of LHCII complexes per liposome (high L:P ratio results in QY similar to that of the unquenched LHCII in detergent micelles). Finally, the fluorescence quenching in these proteoliposomes appears to be driven by protein-protein interactions and not by a specific thylakoid lipid microenvironment ${ }^{31,32}$.

It is important to mention that the typical protocol for LHCII reconstitution into liposomes, utilized in currently reported works, does not entirely rule out the possibility that free (nonreconstituted) LHCII complexes might remain in the sample. This reconstitution protocol relies on detergent removal, and it is known that in low detergent concentrations LHCII forms aggregates ${ }^{33}$. Thus after reconstitution, there may still possibly be the aggregates in the solvent or on the surface, contributing to the observed reduction of the total QY. That would also result in the effective L:P ratio 
in the proteoliposomes being different from the bulk stoichiometry used during the sample preparation, which could explain a large mismatch between the reported magnitude of fluorescence quenching and the L:P ratios ${ }^{30,32}$. Finally, a rather wide distribution in the sizes of the formed liposomes additionally increases the uncertainty of the L:P ratio, thus further complicating the straightforward interpretation of the results obtained during the ensemble-type experiments ${ }^{34}$. Performing density gradient centrifugation and using specific fractions for further analysis ${ }^{35}$ allows one to reduce, but not entirely avoid this uncertainty. Therefore, it is highly critical to develop a method to directly reveal the correct L:P ratio of the proteoliposomes and measure their heterogeneity, thus characterizing the efficiency of LHCII reconstitution into the liposomes.

Here we report a novel method for preparation of LHCII proteoliposomes from native lipid composition that allowed us to address the issues specified above by combining several spectroscopic techniques. First, we employ very sensitive Total Internal Reflection Fluorescence (TIRF) microscopy and novel data analysis methods ${ }^{36}$ to detect single proteoliposomes and characterize the actual L:P ratio and number of LHCII complexes per liposome. Also, we utilize previously published approach combining dynamic light scattering (DLS) and confocal microscopy methods ${ }^{37}$ to characterize the liposome size. A critical methodological characteristic of the present work is the use of a lipophilic dye $^{38}$ as a reporter to label single liposomes. We have also employed improved surface modification

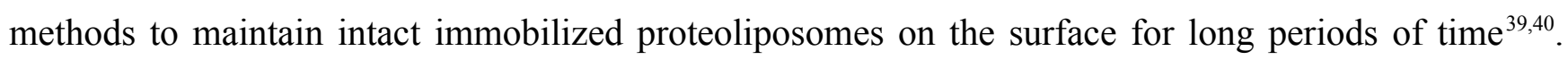
The obtained results clearly indicate that a substantial number of LHCII complexes remain nonreconstituted into the liposomes, suggesting that at least some of the previously reported conclusions on LHCII proteoliposomes might/should be revisited in the future studies. Our reported methods can also be directly applied to study other proteoliposomes on a single-liposome level. 


\section{Materials and Methods}

Isolation of LHCII. LHCII was isolated from 14-day-old pea (Pisum sativum) plants by solubilization of PSII-enriched membranes with n-dodecyl- $\beta$-D-maltoside ( $\beta$-DDM, Cube Biotech, Germany $)^{41,42}$. The LHCII trimer bands were collected and concentrated (Amicon Ultra, Millipore; USA); the Chlorophyll (Chl) concentration was measured according to published procedure ${ }^{43}$ and the samples were frozen in liquid $\mathrm{N}_{2}$ and stored at $-80^{\circ} \mathrm{C}$ until use.

Preparation of liposomes. Liposomes were prepared from plant thylakoid lipids by using previously published protocol ${ }^{10}$ with modifications. A chloroform: methanol solution mixture of isolated plant thylakoid lipids, monogalactosyldiacylglycerol, digalactosyldiacylglycerol, phosphatidylglycerol, and sulfoquinovosyldiacylglycerol (Avanti Polar Lipids, USA) was prepared at a molar ratio of 5:3:1:1. The lipophilic fluorescent dye DiI (Invitrogen, USA) was added to the mixture at a lipid-to-dye ratio of 50:1. Dil was used as a probe to estimate the lipid: protein ratio in reconstituted proteoliposomes. Biotinylated lipid ((1,2-distearoyl-sn-glycero-3-phosphoethanolamine-N-[biotinyl(polyethylene glycol)-2000]) (Avanti Polar Lipids) was added into the mixture at a ratio of 250:1 (lipids : biotinlipid).

The chloroform-methanol lipid mixture was slowly dried in a vacuum rotatory evaporator to form a thin film of lipids on the wall of a round-bottom glass vial. After completely drying the solvent, the lipid film was hydrated with a buffer containing $10 \mathrm{mM}$ Tris-aminomethane (pH 7.8) and $10 \mathrm{mM} \mathrm{NaCl}$ (henceforth termed reconstitution buffer) to a total lipid concentration of $5 \mathrm{mg} / \mathrm{ml}$. The suspension was vortexed for approximately 40-50 min and subsequently subjected to ten freeze-thaw cycles and then extruded through a 100-nm pore membrane (Avanti Polar Lipids, USA). After centrifugation on the ficoll gradient, a sharp band representing empty liposomes was observed, while no free DiI were detected in the supernatant, which demonstrate very high (close to unity) efficiency of the implemented liposome labelling by DiI. 
Reconstituted LHCII membranes. Isolated LHCII complexes were inserted into DiI liposomes to form reconstituted LHCII membranes (proteoliposomes) as previously described ${ }^{10}$ with slight modifications. The DiI liposomes were destabilized by adding $0.05 \%$ detergent $(\beta-\mathrm{DDM})$. The trimeric LHCII complexes, dissolved in $\beta$-DDM were added drop-wise to a suspension of liposomes at concentration of $5 \mathrm{mg} / \mathrm{ml}$, while agitating continuously, to obtain a mixture of desired molar L:P ratio (from 100:1 to $1500: 1$ ). The $\mathrm{L}: \mathrm{P}^{(\mathrm{rm})}$ ratio of this reconstitution mixture was estimated taking into account that LHCII trimer contains 14 Chls per monomer. The lipid-protein mixture was incubated at room temperature for $30 \mathrm{~min}$ in dark. The detergent was then removed by repeated incubation of the sample with absorbent beads (Bio-Beads SM2, Bio-Rad, USA) - a total of $240 \mathrm{mg} / \mathrm{ml}$ beads were added in three $80 \mathrm{mg}$ portions incubating for 1 hour, overnight and 2 hours, respectively. This method removes almost all detergent in the suspension ${ }^{44}$. The samples were further diluted several-fold during the next purification steps, so any remaining free detergent was eventually removed.

Separation of proteoliposomes by density. Proteoliposome fractions of different density and lipid: protein ratios were separated by density gradient ultracentrifugation. Discontinuous (step) gradients were prepared by adding $2 \mathrm{ml}$ layers of 7, 14, 21, and 28\% Ficoll PM-400 (GE Healthcare, USA) dissolved in reconstitution buffer. The proteoliposome sample was loaded on the gradient and centrifuged for 18 hours at $200,000 \mathrm{xg}, 4^{\circ} \mathrm{C}$. Four to five colored band fractions of different densities were collected.

\begin{abstract}
Absorption spectroscopy. Absorption spectra in the visible region were recorded at room temperature with a Nicolet Evolution 500 dual-beam UV-VIS spectrophotometer (Thermo Fisher Scientific, USA). The sample and reference solution were placed in 1-cm path length optical glass cuvettes and absorption was measured with $1 \mathrm{~nm}$ spectral resolution. The spectra were analyzed and graphed using the Spectr-O-Matic software.
\end{abstract}

Quantification of the L:P ratio in bulk. To quantify the L:P ratio in bulk (SI Table 2), first we determined the apparent molar absorption coefficients of Chl in LHCII and of DiI in control liposomes 
at $674 \mathrm{~nm}$ and $553 \mathrm{~nm}$. The apparent Chl absorption coefficients were determined from the absorption spectra of LHCII of known total Chl concentration $(\mathrm{Chl} a+b)$, which were estimated from pigment extracts in $80 \%$ acetone (SI Table 1$)^{43}$. The molar concentration of Chl $(a+b)$ and DiI in the LHCII proteoliposomes was estimated from absorption spectra using the following formula: $C_{\mathrm{Chl}}=A_{674} / \epsilon_{674}^{\prime}$, $C_{\text {DiI }}=\left(A_{553}-\epsilon_{553}^{\prime} \cdot C_{\mathrm{Chl}}\right) / \epsilon^{\prime \prime}{ }_{553}$, where $\mathrm{A}_{553}$ and $\mathrm{A}_{674}$ are the measured absorbances and $\epsilon^{\prime}$ and $\epsilon^{\prime \prime}$ are the apparent molar absorption coefficients of Chl and DiI, respectively (SI Table 1). The molar L:P ratio = $\left(C_{\mathrm{Dil}} \cdot \mathrm{LDR}\right) /\left(C_{\mathrm{Chl}} / 14\right)$, here LDR is the lipid-to-DiI ratio of the liposomes and 14 is the number of Chls per LHCII monomer.

Immobilization of proteoliposomes for microscopy experiments. Silanized and PEGylated (methoxy-PEG and biotin-PEG) glass slides (\#1.5, Menzel Glaser) were prepared in the same way as described previously ${ }^{45}$. These glass slides were assembled into the 6-channel flow cells (sticky-Slide VI 0.4, IBIDI, Germany). Next channel was filled with the reconstitution buffer $(300 \mu 1)$ and incubated with Neutravidin (nAv, A-26666, Molecular probes) solution $(0.02 \mathrm{mg} / \mathrm{ml})$ for minimum 3 minutes. Free nAv was removed by washing the channel with the reconstitution buffer $(300 \mu l)$. Next proteoliposomes were injected into the channel at low concentration and incubated until sufficient liposome density was achieved. Free proteoliposomes were removed by washing the channel with the reconstitution buffer $(300 \mu 1)$. After this step sample was ready for microscopy measurements. During fluorescence microscopy measurements liposomes were immobilized and washed thoroughly with detergent-free buffer. Therefore there was low probability to have detergent in the solution during the microscopy experiment.

Microscopy measurements. We have employed TIRF microscopy and confocal microscopy setups to collect various information of the obtained LHCII proteoliposome samples. TIRF microscopy was used to analyze the co-localization of liposomes and LHCII complexes: this method does not involve intensity characterization of the observed fluorescent spots, but instead checks for spatial overlap of the 
spots detected in the DiI and LHCII channels (Fig. 2d).. Being a fast technique allowing one to collect sufficient statistics with good signal-to-noise ratio, TIRF microscopy was also used to estimate the number of LHCII per liposomes from the bleaching traces of the LHCII complexes. Meanwhile, confocal setup provides even excitation of different size liposomes and therefore it was employed to characterize liposome sizes and protein densities.

TIRF microscopy. Our home-build setup was previously described ${ }^{22,45}$. For these measurements beam expander, mounted before the focusing lens, was changed into $7 \mathrm{x}$, which allowed us to optimize the evenness of the illumination field. Laser power before the objective was set to $2.5 \mathrm{~mW}$ for both $532 \mathrm{~nm}$ and $632 \mathrm{~nm}$ lasers (20mW both, Crystalazer, USA). That resulted in laser power density of $\sim 12 \mathrm{~W} / \mathrm{cm}^{2}$ at the plane of the sample. Exposure time of the EMCCD camera (Ixon3 Andor) was set to $50 \mathrm{~ms}$. In the TIRF microscopy images we have detected no signal of DiI in the LHCII channel under $532 \mathrm{~nm}$ excitation and also no signal of LHCII in the DiI channel under $635 \mathrm{~nm}$ excitation (data not shown). The penetration depth of the evanescent field was set to $\sim 300 \mathrm{~nm}$ for both wavelengths of excitation.

Confocal microscopy. Our home build confocal microscope uses the same excitation part as the TIRF microscope, except that for the red channel excitation we have used a pulsed diode laser $(635 \mathrm{~nm}$ wavelength, 50MHZ, Becker \& Hickl, Germany). Laser power at the plane of the sample was set to $\sim 0.21 \mu \mathrm{W}\left(10 \mathrm{~W} / \mathrm{cm}^{2}\right)$ for the red laser and $\sim 0.9 \mu \mathrm{W}\left(180 \mathrm{~W} / \mathrm{cm}^{2}\right)$ for the green laser. The fluorescence emission was collected with the same objective as in TIRF (100x, 1.4NA, Nikon), passed through fourband emission filter (446/510/581/703 nm, Semrock, USA), and then was directed to the dichroic mirror where the light was split into green and red components. The intensity of the green component was measured with one APD (tau-SPAD, PicoQuant, Germany) and intensity of the red componentwith a second identical APD. Intensity counts from the APDs were readout by a NI card (SCB-68, NI, USA) connected to the FPGA card (PCIe-7851R, NI, USA). Sample was scanned with the piezo stage (P-733.2CD, PI, Germany) with the controller (E-712.3CDA, PI, Germany). Control of the scanning 
and read out was achieved by custom written Lab-View (NI, USA) software. Pixel dwell time was set to $5 \mathrm{~ms}$, size of the image was set to $200 \times 200$ pixels.

Image analysis. Image analysis was performed using custom-written software (Igor Pro 6.37, USA) as described previously ${ }^{22,45}$. To extract the integrated fluorescence intensity, the intensity of the detected spots was fitted with a two-dimensional symmetric Gaussian function. To calculate the number of LHCII trimers per liposome we analyzed their photobleaching traces as described previously ${ }^{36}$. Briefly, bleaching movies of LHCII under continuous excitation with $635 \mathrm{~nm}$ laser were acquired in TIRF mode. In these movies we have detected fluorescent spots and extracted the bleaching traces (see Supplementary Fig. 2). The number of LHCII molecules per bleaching trace was calculated using the recently published model fitting algorithm ${ }^{36}$. It must be noted that although single LHCII trimer exhibits multiple fluorescence intensity levels changing over time ${ }^{18,19,46}$, its bleaching is a single stepprocess. Also, individual bleaching steps, recorded in bleaching traces under the described imaging conditions, are short-lasting, and protein does not show much fluorescence blinking before it bleaches out. Therefore, although the fluorescence intensity fluctuations complicate the analysis, the algorithm accounts just for the difference in the mean fluorescence intensity between individual bleaching steps in the bleaching trace, thus enabling us to correctly evaluate the number of LHCII per liposome. Another aspect of the photobleaching analysis is its assumption that every fluorophore was subjected to similar excitation intensity. This assumption complicates the analysis because in larger liposomes due to the exponentially decaying excitation field in the $\mathrm{Z}$ direction, LHCIIs located in different parts of the liposome may be exposed to different excitation intensities. Therefore, the estimated number of proteins per liposome has some uncertainty that approximately exponentially depends on the liposome size. For the liposomes that are smaller than $100 \mathrm{~nm}$ in diameter, this uncertainty is less than $10 \%$, and for those that are $\sim 300 \mathrm{~nm}$ in diameter, it reaches $\sim 30 \%$. Thus this uncertainty becomes significant only for the liposomes whose size is similar to or larger than the penetration depth of TIRF, and in our 
samples, the majority of proteoliposomes are below this value. Also our method enables selection of proteoliposomes based on their size, therefore one can analyze only those proteoliposomes, that are below the penetration depth of TIRF system.

DLS and size calibration. To convert liposome intensities into diameter in $\mathrm{nm}$ we have followed previously described procedure ${ }^{37}$. We have measured average liposome sizes of the control liposome sample without protein, that was prepared in the same way as proteoliposomes, using DLS (Zetasizer $\mu \mathrm{V}$, Malvern Analytical). Our control liposomes had average diameter of $106 \mathrm{~nm}$. On our microscope setups we have imaged the same control liposomes as in DLS, obtained integrated intensities, took square root of the intensities and calculated average of these distributions. The average diameter obtained from DLS and average square root intensity allowed us to obtain numeric conversion factor of 10.5 to convert from fluorescence intensity of liposome into diameter in $\mathrm{nm}$.

We note that, although being high, DiI labeling efficiency is not identical in all liposomes and might exhibit up to $\sim 30 \%$ error, which however strongly depends on the lipid used ${ }^{28}$. This effect gives a particular uncertainty for the liposome size calculation, which previously was estimated to be around $20 \%$, and, due to the Poisson noise of the small number of chromophores is more pronounced for the small liposomes ${ }^{28,47}$. However there is no good way to precisely correct for this minor effect, and therefore we did not perform any additional correction for determined liposome size.

For TIRF microscopy the liposomes sizes were determined in the same way as with the confocal microscopy. It must be noted that TIRF microscopy might produce a somewhat biased estimation of the liposome diameter because of the excitation unevenness in the direction perpendicular to the glass surface $^{48}$. Nevertheless, we observed a similar trend results obtained by both microscopy types.

\section{Results}

Bulk spectroscopy. Reconstitution of the LHCII complexes into liposomes is a rather complicated process involving detergent removal from the solution comprising solubilized pigment-protein 

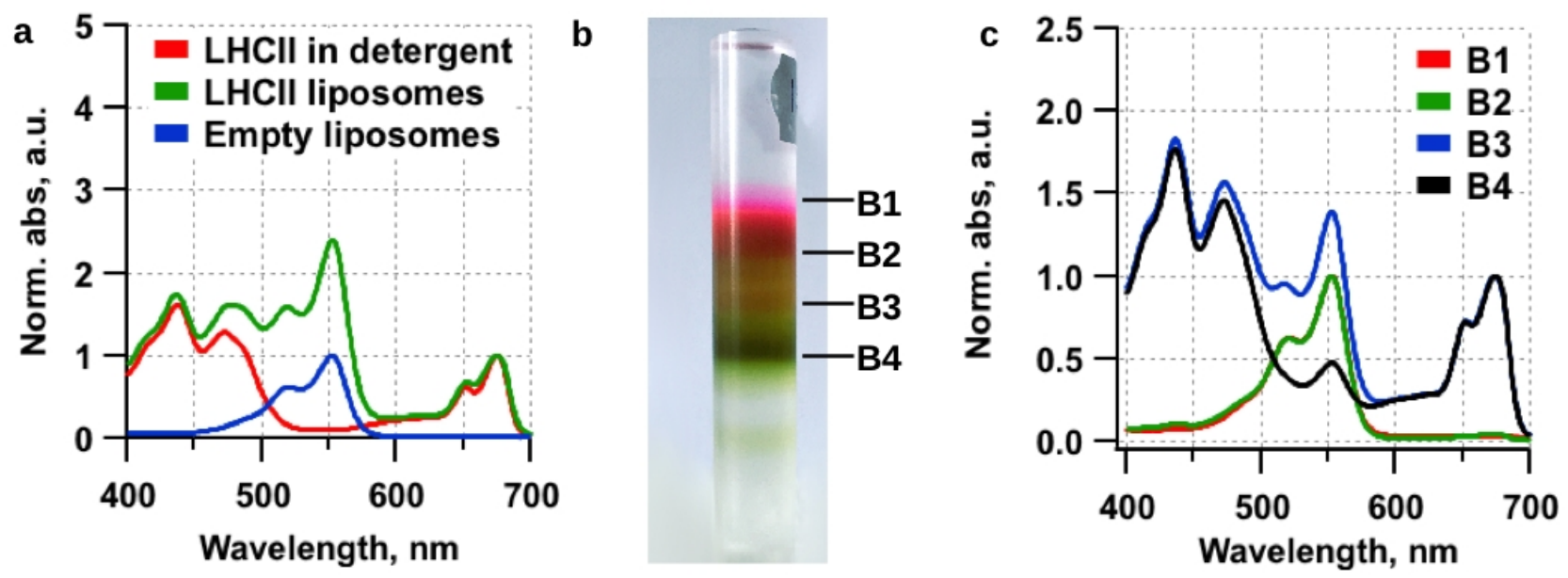

Figure 1 | LHCII proteoliposome fractions and their absorption spectra. a, Absorption spectra of control liposomes, LHCII trimers in detergent micelles, and LHCII proteoliposomes at L:P ratio of the reconstitution mixture being 500:1. The latter two spectra are normalized at $674 \mathrm{~nm}$. b, Separation of LHCII liposome fractions by gradient density. Differently colored bands of increasing density in ficoll are denoted as B1-B4. c, Absorption spectra of proteoliposome sample bands B1-B4 from reconstitution mixture with L:P ratio of 1500:1. The spectra of B3-B4 bands are normalized at $674 \mathrm{~nm}$.

complexes and liposomes. As a result, two competing processes are initiated-LHCII aggregation within the solution ${ }^{49}$ and LHCII insertion into the liposomes ${ }^{50}$. Therefore before performing any detailed analysis of the obtained data, it is critical to determine the reconstitution level and evaluate the correct L:P ratio in the produced proteoliposomes.

LHCIIs are trimeric complexes comprising 42 chlorophyll (Chl) pigments, and therefore they are naturally fluorescent. Contrarily, liposomes do not exhibit any fluorescence signal and are not suitable for the direct quantitative spectroscopic analysis. To overcome this issue, we have employed the lipophilic stain DiI (see Supplementary Fig. 1) during the liposome production. DiI is a very stable membrane dye that due to its spectral characteristics is perfectly suitable for labeling LHCII proteoliposomes. Indeed, the absorption spectrum of LHCII complexes is characterized by two absorption bands (Fig.1a). The 650-700 nm wavelength region manifests the strong $Q_{y}$ transitions of chlorophylls (the Chl $a$ and Chl $b$ pigments). The absorption band in the $400-500 \mathrm{~nm}$ spectral region corresponds to the Soret band of the Chls and the absorption band of the carotenoid pigments ${ }^{1}$. Meanwhile, the DiI dyes in the control liposomes (prior to LHCII reconstitution) exhibit a strong 
absorption band around $550 \mathrm{~nm}$ - exactly at the minimum of the LHCII absorption spectrum (Fig. 1a). The absorption spectrum of LHCII proteoliposomes, formed by using the 500:1 lipid-to-protein ratio of the reconstitution mixture (denoted below as $\mathrm{L}: \mathrm{P}^{(\mathrm{rm})}$ ), is also presented in Fig. 1a and clearly exhibits the additive nature of its sub-bands.

The reconstitution of protein into liposomes depends on many factors ${ }^{50}$, and typically proteins are not evenly incorporated ${ }^{34,51}$. Depending on the reconstitution conditions, there could remain empty liposomes (those without LHCII complexes) as well as free LHCIIs (either aggregated, non-aggregated, or both). As the first step to separate empty liposomes and free LHCIIs from proteoliposomes, we performed density gradient ultracentrifugation. Density gradients were prepared with Ficoll 400 that is a high molecular weight polymer of sucrose having low osmotic pressure to keep the liposomes intact.

After centrifugation, several gradient bands of different color were observed and collected as separate fractions (see Fig 1b). The lowest-density gradient band (B1 in Fig. 1b) had a distinct pink color, showed the absorption spectrum of pure DiI (peaking at $553 \mathrm{~nm}$ ) without any Chl (at $674 \mathrm{~nm}$ ), indicating that it consists entirely of empty liposomes (Fig. 1c). This fraction was excluded from further analysis. Bands of higher density (B2-B4) had orange-brown to green color and exhibited increasing relative absorption in the $\mathrm{Chl}$ region at the expense of DiI absorption, indicating progressively higher protein and lower lipid content. That could correspond to either lower abundance of unoccupied liposomes or lower L:P content of the proteoliposomes.

The differences in the molar absorption coefficients of the Chls and DiI dyes at various wavelengths (shown in Supplementary Table 1) allowed us to estimate the mean lipid-to-protein ratio in these distinct gradient bands $\left(\mathrm{L}: \mathrm{P}^{(\mathrm{gb})}\right)$, see Methods for details. By varying the lipid-to-protein ratio of the initial reconstitution mixture $\left(\mathrm{L}: \mathrm{P}^{(\mathrm{rm})}\right)$, we found that in all the samples the $\mathrm{L}: \mathrm{P}^{(\mathrm{gb})}$ ratio of the $\mathrm{B} 2$ was about 2-3 times larger than that of the initial mixture (see Supplementary Table 2). Meanwhile, the $\mathrm{L}: \mathrm{P}^{(\mathrm{gb})}$ ratio of the higher-density bands was spread over broad range of values. In most cases the fractions containing the largest amount of Chl had L:P(gb) considerably lower than the corresponding 
$\mathrm{L}: \mathrm{P}^{(\mathrm{rm})}$. Only rarely the two quantities were of similar magnitude, and there was no clear correlations between them. As a result, there is no practical way to predict the L:P ratio in the proteoliposomes a priori by varying the concentration of the reconstitution mixture. Moreover, even the separation of the proteoliposomes into distinct gradient bands does not entirely rule out the possibility of coexistence of empty liposomes and free LHCIIs in the same band.

TIRF microscopy of single proteoliposomes. To address the issue of possible heterogeneity of the LHCII proteoliposomes and to characterize the reconstitution efficiency, we have used fluorescence microscopy and employed proteoliposome anchoring strategy on a surface at low densities (shown schematically in Fig. 2a) 34,39,40,52,53. Particularly, during the production of the liposomes, we have

a
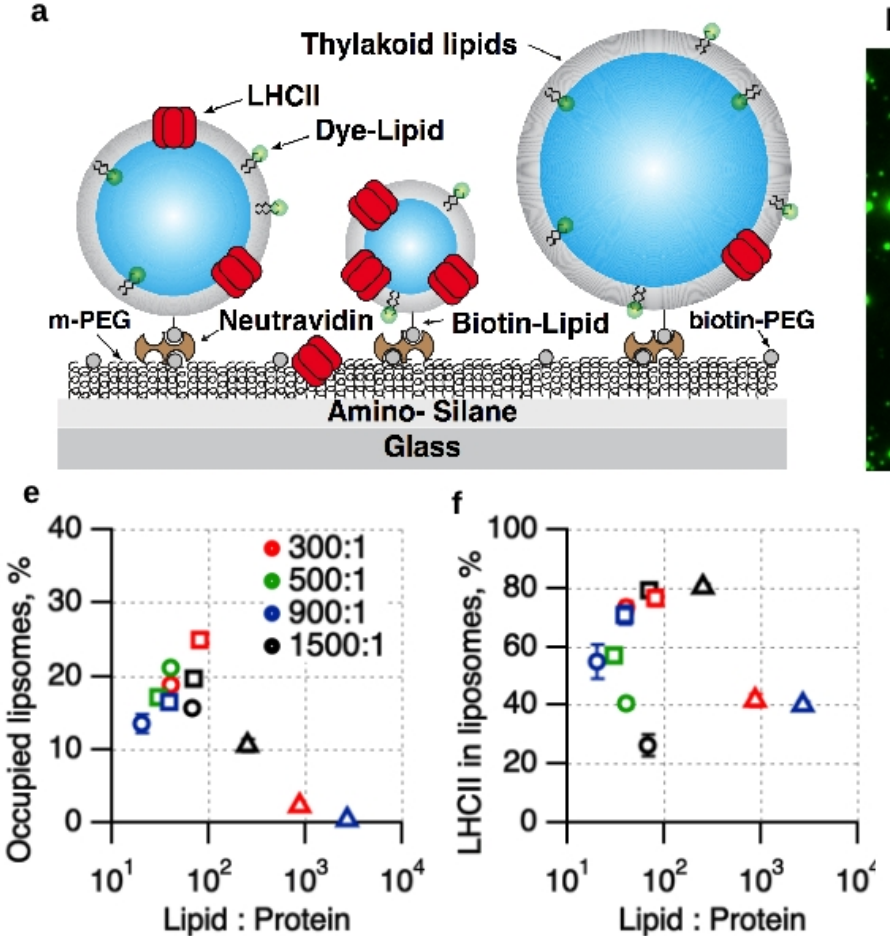
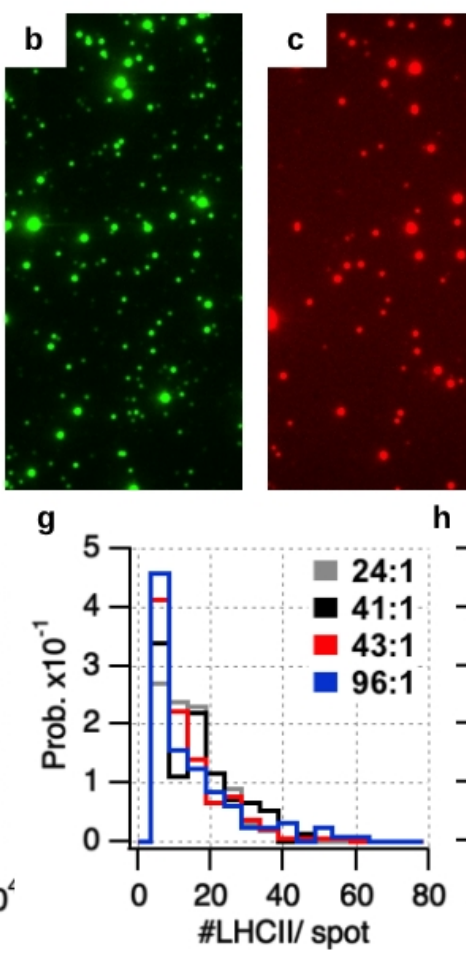

h
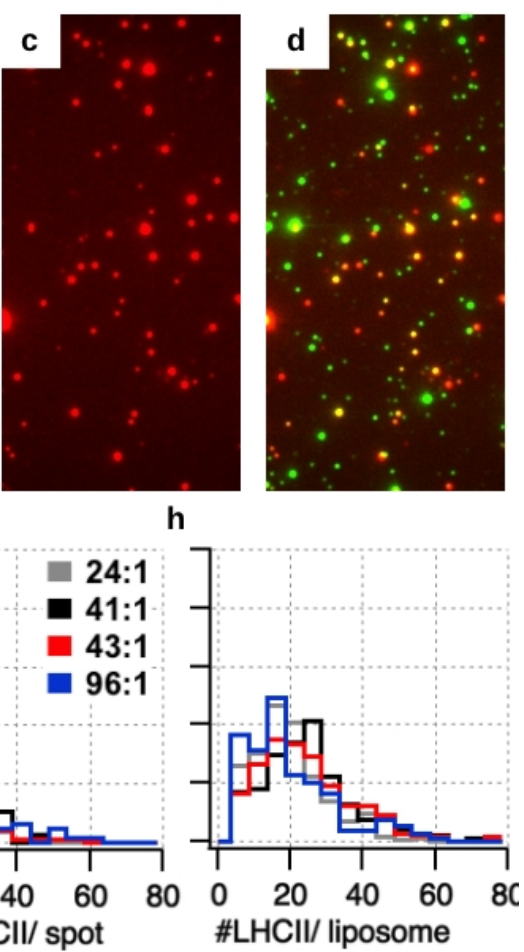

Figure 2 | TIRF microscopy of single LHCII proteoliposomes. a, The experimental scheme showing immobilization of the variously-sized proteoliposomes that contain both DiI dyes and biotin-lipids on the silanized and PEGylated (10\% biotin-PEG) glass coverslip via Neutravidin. b-d, TIRF images obtained via the DiI channel at $532 \mathrm{~nm}$ excitation (b), LHCII channel at $635 \mathrm{~nm}$ (c) and both channels overlaid (d). e, Percentage of the occupied liposomes vs. lipid/protein ratio of the gradient band. Colors indicate L:P(rm) of the reconstitution mixture, symbols - different gradient bands: B2 (triangles), B3 (squares) and B4 (circles). f, Percentage of reconstituted LHCII complexes vs. L: $\mathrm{P}^{(\mathrm{gb})}$ of the gradient band. Color in symbol notation is the same as for panel e. $\mathbf{g}-\mathbf{h}$, The statistical distribution of the number of LHCII complexes per spot representing the free surface-bound LHCIIs (g) and per liposome (h) in the TIRF image, calculated from bleaching traces for four distinct $\mathrm{L}: \mathrm{P}^{(\mathrm{gb})}$ ratios shown in the legend. 
included the biotinylated lipids that served as an anchor of the liposomes onto the silanized and PEGylated glass surface via Neutravidin (see Materials and Methods for details). Under these anchoring conditions, liposomes remain intact and maintain permeability to ions ${ }^{39}$. Thus they still reflect the natural membrane conditions for the embedded proteins. Besides that, the mentioned glass surface modification ensures low non-specific binding of both liposomes and proteins.

For the measurements, we have employed TIRF microscopy that provided high sensitivity and the possibility to image the massive amount of proteoliposomes in a parallel manner. In these experiments, we recorded images of two fluorescence channels (red for LHCII and green for DiI) under different excitation conditions: excitation of DiI dyes at $532 \mathrm{~nm}$ wavelength and excitation of LHCII at $635 \mathrm{~nm}$ (see Fig. 2b-d). Next, the fluorescent diffraction-limited spots observed in the acquired images were analyzed using custom-written automated software to detect the co-localized spots. This procedure allowed distinguishing the spots that correspond to the proteoliposomes containing successfully embedded LHCII complexes (when both signals coincide) from just empty liposomes (no signal in the Chl channel) or non-reconstituted LHCIIs that were non-specifically bound to the surface (no signal in the DiI channel).

The performed co-localization analysis revealed that no gradient bands were devoid of empty liposomes or non-reconstituted protein. The percentage of liposomes incorporating LHCII was rather low in all of the examined samples and does not exceed $25 \%$ of the total number of liposomes (see Fig. 2e and Supplementary Table 3). In higher density bands (B3, B4), it increases with the L:P(gb) ratio (albeit exhibits no general trend with the $\mathrm{L}: \mathrm{P}^{(\mathrm{rm})}$ ) and reaches the maximal value of $25 \%$ at $\mathrm{L}: \mathrm{P}^{(\mathrm{gb})} \approx 100: 1$. We see therefore that, counterintuitively, the gradient bands with the lower L:P (thus higher amount of LHCII) exhibit substantially higher percentage of the empty liposomes. A rather low percentage of occupied liposomes was observed in the low-density gradient band (B2), which confirms our previous assumption based on the absorption spectrum. 
In addition to the percentage of LHCII incorporating liposomes, the co-localization analysis also provided us a piece of information about the surface-bound free LHCII. Notably, we found that in some gradient bands up to $75 \%$ of fluorescing spots detected in the LHCII channel images correspond to the non-reconstituted LHCIIs (see Fig. 2f and Supplementary Table 3). Overall, in different samples, the efficiency of the LHCII incorporation into the liposomes varied between $25 \%$ and $80 \%$, but no strict dependency on neither $\mathrm{L}: \mathrm{P}^{(\mathrm{gb})}$ nor $\mathrm{L}: \mathrm{P}^{(\mathrm{rm})}$ was observed. In the similar studies of $\beta 2 \mathrm{AR}$ protein, it was demonstrated previously that by using this method of liposome labeling it is impossible to observe such a vast amount of free proteins because of merely insufficient liposome labeling ${ }^{34}$. These results, therefore, demonstrate the importance of our employed co-localization assay, as it allows direct monitoring of the LHCIIs embedded into the liposomes.

By analyzing the fluorescence bleaching traces of the spots from TIRF microscopy images corresponding to the LHCII complexes, we were able to evaluate the number of the LHCII trimers fluorescing at that specific spot (assuming no excitation energy transfer between LHCIIs within the same spot, see Materials and Methods for details). We obtained statistical distributions of the cluster sizes for several distinct $\mathrm{L}: \mathrm{P}^{(\mathrm{gb})}$ ratios, which revealed that the free surface-bound LHCII complexes formed mostly small clusters comprising about 10-13 LHCII trimers per each spot, albeit several larger aggregates of up to 50 LHCIIs were also detected (Fig. 2g). On the other hand, the number of LHCII trimers embedded into the liposome exhibited a much wider distribution, varying from just a few complexes up to 80 trimers per liposome for different spots (see Fig. 2h). In principle, this broad distribution indicates either widespread of liposome sizes or heterogeneous distribution of the LHCII density in various liposomes or both.

Confocal microscopy of single proteoliposomes. To access the liposome sizes in nanometers, we performed confocal microscopy studies of the same samples as in the previously described TIRF microscopy, measuring the two fluorescence intensity signals following excitation at $532 \mathrm{~nm}$ (DiI dyes) and $635 \mathrm{~nm}$ (LHCII complexes); see Supplementary Fig. 3 for the obtained images. Again, automated 
spot detection software allowed us to perform the co-localization of the liposomes and LHCIIs analysis. By measuring the fluorescence intensity of control empty liposomes (see Supplementary Fig. 4) and comparing their intensity distributions with the dynamic light scattering data of the same sample, we were able to calibrate the measurement results and convert the liposome's fluorescence intensity into its diameter expressed in $\mathrm{nm}^{37,40}$. The same conversion factor was also used for other samples containing proteoliposomes.

The ratio of fluorescence intensities of LHCII and liposome dyes at each co-localized spot, reflecting the protein density in a particular detected single liposome, demonstrated a strong dependence on the liposome size (Fig. 3a). The protein density in the individual proteoliposome exhibited a broad distribution for liposomes smaller than $200 \mathrm{~nm}$ in diameter, and reduced severely in larger liposomes. On the other hand, as expected, the evaluated mean protein density of the proteoliposomes scaled linearly with the inverse of the $\mathrm{L}: \mathrm{P}^{(\mathrm{gb})}$ ratio of the corresponding gradient band (Fig. 3b). Meanwhile, the mean diameters of the proteoliposomes detected in different gradient bands did not exhibit any correlation with the $\mathrm{L}: \mathrm{P}^{(\mathrm{gb})}$ ratio of that band. It is also noteworthy to mention that both liposome sizes and protein density per liposome demonstrated a very inhomogeneous distribution within each gradient band, indicating that the spectroscopic information obtained from ensemble
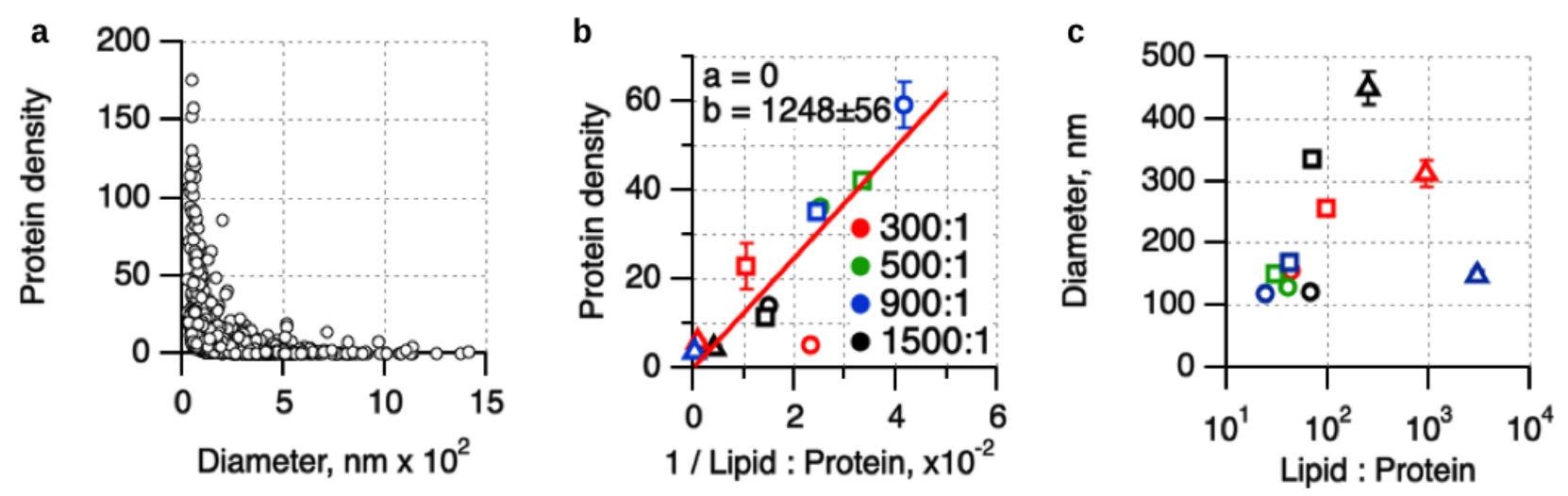

Figure 3 | Confocal microscopy of single LHCII proteoliposomes. a, Relation between the protein density in the proteoliposome (the ratio of fluorescence intensity of LHCII and liposome dyes) and the liposome diameter. $\mathbf{b}$, Linear correlation between the mean protein density in the proteoliposome and the inverted $\mathrm{L}: \mathrm{P}^{\mathrm{(gb})}$ ratio of the gradient band. c, Average liposome diameter vs. L:P of the gradient band. 


\begin{abstract}
measurements on the proteoliposomes might be not sufficient for proper interpretation, and treating of both parameters on a single-liposome level is highly critical.
\end{abstract}

\title{
Discussion
}

Reconstitution of membrane proteins into liposomes is a widely used approach to study the structure and function of various proteins in the conditions mimicking natural environment. In this work, we have demonstrated that the usage of the lipophilic DiI dye during the preparation of LHCII proteoliposomes has proven to be a suitable method for labelling liposomes. It allowed them to be studied at the single-liposome level using TIRF and confocal microscopy. By implementing TIRF microscopy we addressed efficiency of LHCII reconstitution into liposomes while by applying confocal microscopy, we were able to quantify the size of individual liposomes and the relative protein density therein. Particularly, the obtained results revealed that each of the density bands, which was supposed to be purified to some extend from free LHCII, still remained a highly heterogeneous mixture of empty liposomes, variously-sized proteoliposomes, and free LHCIIs. We found no gradient band consisting entirely of proteoliposomes. In any of given gradient density band only up to $25 \%$ of all liposomes incorporated LHCII, as well as at least $20 \%$ of the LHCII complexes formed clusters with no detectable lipid. Also, judging from the bulk fluorescence lifetime results and the CD spectra (SI Fig. 5), there were no evidences for remaining detergent solubilized LHCII (no $\sim 4$ ns lifetime, and no aggregation signature bands at $436 \mathrm{~nm}$ and $484 \mathrm{~nm}$ ) and for LHCII monomerization (loss of CD amplitude at 473 $\mathrm{nm}$ ) found in samples, which were used for microscopy. This implies severe difficulties while interpreting bulk spectroscopic measurements when the signals from all these subpopulations are mixed, suggesting that some previously reported results might have to be revisited. 
The single-vesicle TIRF microscopy analysis enabled us also to determine another important structural parameter - the protein surface concentration, i.e. the absolute number of the LHCII trimers per unit surface area of the liposome (see Materials and Methods for details). Although exhibiting wide scatter, this parameter showed striking dependence on the liposome diameter - smaller liposomes tended to demonstrate higher protein concentration, see Fig. 4. This trend spans over almost six orders of magnitude and exhibits a power-law dependence. Interestingly, in all of the examined samples, the exponent of this power-law dependence was close to -2 , which means that the absolute number of LHCII complexes per liposome is independent of the liposome diameter. Indeed, a wide scatter of the data points in Fig. 4 reveals that the reconstituted proteoliposomes with statistically almost equal probability can contain from several to $\sim 50$ LHCII complexes. Such behavior might seem as a rather surprising result contradicting the intuitive assumption that larger liposomes should on average accumulate a larger number of the protein complexes. It is expected, however, that the balance between the elastic and hydrophobic forces acting on the LHCII complexes, embedded into lipid bilayer, thermodynamically favors their reconstitution into the small liposomes rather than into the larger ones $^{54}$. The combined effect of these two opposing factors indeed results in our observation that the

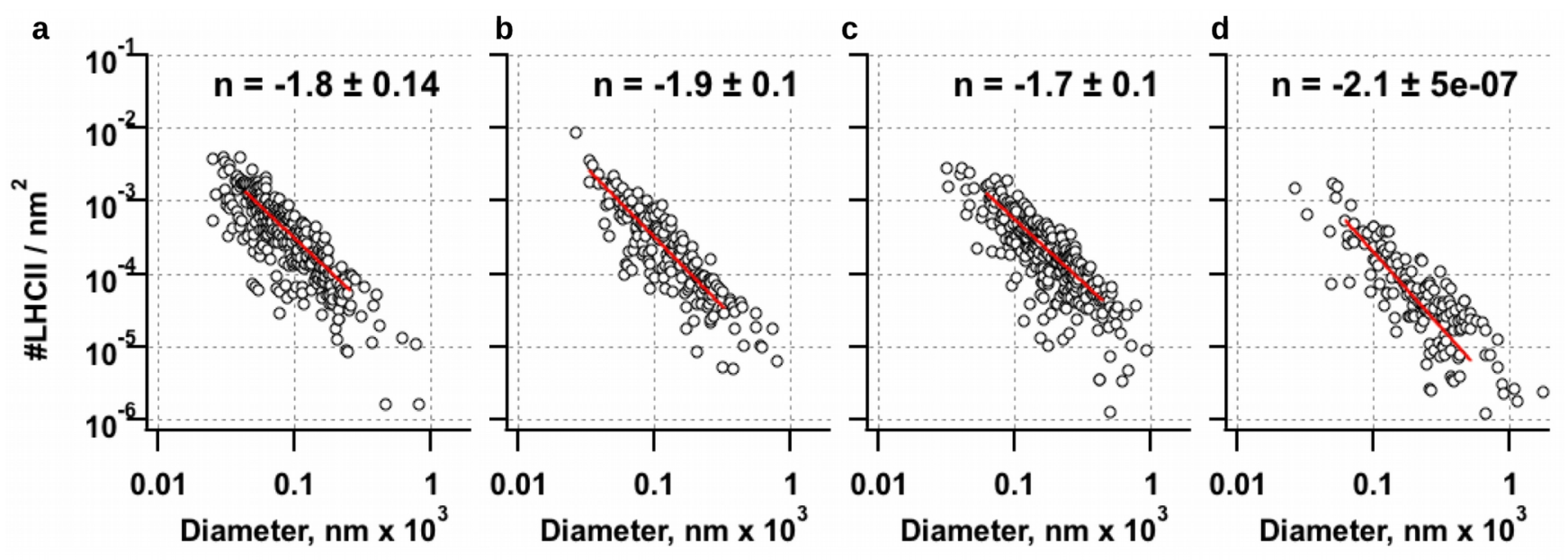

Figure 4 | Distribution of the LHCII mean surface concentration (the number of LHCII trimers per unit surface area of the proteoliposome) for variously sized liposomes. The panels correspond to different lipid/protein ratios of the B3 and B4 gradient bands from different reconstitution mixtures: L:P(gb) = 24:1 (a), 41:1 (b), 43:1 (c), and 96:1 (d). Red lines demonstrate the power-law description, $\mathrm{y} \propto \mathrm{x}^{\mathrm{n}}$, and the exponent $\mathrm{n}$ is indicated in each case. 
number of the LHCII complexes incorporated into the liposome in principle does not depend on the liposome size or the $\mathrm{L}: \mathrm{P}^{(\mathrm{gb})}$.

To summarize, we have shown quantitatively that the liposome formation and subsequent reconstitution of the LHCII complexes into them result in the by-all-means heterogeneous systems: in terms of the liposome size, the number of pigment-protein complexes embedded into individual proteoliposomes and, consequently, the protein surface density. More importantly, the resulting L:P ratio in these proteoliposomes cannot be predicted a priori by varying sample preparation conditions. We have also determined that the process of reconstitution itself is not efficient: at least $75 \%$ of the liposomes remained empty in different gradient bands while, at the same time, from $20 \%$ up to $75 \%$ of the LHCII complexes remained liposome-free. The spectroscopic signal of the latters might severely interfere with one coming from proteoliposomes, complicating therefore the interpretation of the results of simple bulk measurements.. In contrast, liposome labelling with specific dyes and the application of the single-molecule techniques provide a unique tool to clarify this uncertainty and study membranerelated proteins and their function in a controlled native-like environment.

\section{Supporting Information is available}

Bulk characterization results, illustrative images, and other materials.

\section{Acknowledgments}

This work was supported by Lithuanian-Ukrainian grant (P-LU-18-64) and by a grant from the Hungarian Ministry for National Economy (GINOP-2.3.2-15-2016-00001) and the National Research, Development and Innovation Office (NKFIH NN-124904). We thank D. Rutkauskas for help on setting up the confocal microscope and participation in the initial discussion on the project; M. Vengris for lending hardware and help with the signal acquisition software; O. Venckus and I. Čiplys for participation in performing several TIRF and confocal microscopy measurements. 


\section{References}

(1) Blankenship, R. E. Molecular Mechanisms of Photosynthesis; Blankenship, R. E., Ed.; Blackwell Science Ltd: Oxford, UK, 2002.

(2) Horton, P.; Ruban, A. V; Walters, R. G. Regulation of Light Harvesting in Green Plants. Annu. Rev. Plant Physiol. Plant Mol. Biol. 1996, 47 (1), 655-684.

(3) Ruban, A. V.; Johnson, M. P.; Duffy, C. D. P. The Photoprotective Molecular Switch in the Photosystem II Antenna. Biochim. Biophys. Acta - Bioenerg. 2012, 1817 (1), 167-181.

(4) Duffy, C. D. P.; Valkunas, L.; Ruban, A. V. Light-Harvesting Processes in the Dynamic Photosynthetic Antenna. Phys. Chem. Chem. Phys. 2013, 15 (43), 18752.

(5) Haferkamp, S.; Haase, W.; Pascal, A. A.; van Amerongen, H.; Kirchhoff, H. Efficient Light Harvesting by Photosystem II Requires an Optimized Protein Packing Density in Grana Thylakoids. J. Biol. Chem. 2010, 285 (22), 17020-17028.

(6) Johnson, M. P.; Brain, A. P. R.; Ruban, A. V. Changes in Thylakoid Membrane Thickness Associated with the Reorganization of Photosystem II Light Harvesting Complexes during Photoprotective Energy Dissipation. Plant Signal. Behav. 2011, 6 (9), 1386-1390.

(7) Kiss, A. Z.; Ruban, A. V.; Horton, P. The PsbS Protein Controls the Organization of the Photosystem II Antenna in Higher Plant Thylakoid Membranes. J. Biol. Chem. 2008, 283 (7), 3972-3978.

(8) Akhtar, P.; Lingvay, M.; Kiss, T.; Deák, R.; Bóta, A.; Ughy, B.; Garab, G.; Lambrev, P. H. Excitation Energy Transfer between Light-Harvesting Complex II and Photosystem i in Reconstituted Membranes. Biochim. Biophys. Acta - Bioenerg. 2016, 1857 (4), 462-472.

(9) Farooq, S.; Chmeliov, J.; Wientjes, E.; Koehorst, R.; Bader, A.; Valkunas, L.; Trinkunas, G.; van Amerongen, H. Dynamic Feedback of the Photosystem II Reaction Centre on Photoprotection in Plants. Nat. Plants 2018, 4 (4), 225-231.

(10) Akhtar, P.; Dorogi, M.; Pawlak, K.; Kovács, L.; Bóta, A.; Kiss, T.; Garab, G.; Lambrev, P. H. Pigment Interactions in Light-Harvesting Complex II in Different Molecular Environments. $J$. Biol. Chem. 2015, 290 (8), 4877-4886.

(11) Borch, J.; Hamann, T. The Nanodisc: A Novel Tool for Membrane Protein Studies. Biol. Chem. 2009, 390 (8), 805-814.

(12) Bell, A. J.; Frankel, L. K.; Bricker, T. M. High Yield Non-Detergent Isolation of Photosystem ILight-Harvesting Chlorophyll II Membranes from Spinach Thylakoids: Implications for the Organization of the PS I Antennae in Higher Plants. J. Biol. Chem. 2015, 290 (30), 1842918437. 
(13) Liguori, N.; Roy, L. M.; Opacic, M.; Durand, G.; Croce, R. Regulation of Light Harvesting in the Green Alga Chlamydomonas Reinhardtii: The c-Terminus of Lhcsr Is the Knob of a Dimmer Switch. J. Am. Chem. Soc. 2013, 135 (49), 18339-18342.

(14) Zhou, F.; Liu, S.; Hu, Z.; Kuang, T.; Paulsen, H.; Yang, C. Effect of Monogalactosyldiacylglycerol on the Interaction between Photosystem II Core Complex and Its Antenna Complexes in Liposomes of Thylakoid Lipids. Photosynth. Res. 2009, 99 (3), 185-193.

(15) Yang, C.; Boggasch, S.; Haase, W.; Paulsen, H. Thermal Stability of Trimeric Light-Harvesting Chlorophyll a/b Complex (LHCIIb) in Liposomes of Thylakoid Lipids. Biochim. Biophys. Acta Bioenerg. 2006, 1757 (12), 1642-1648.

(16) Wardak, A.; Brodowski, R.; Krupa, Z.; Gruszecki, W. I. Effect of Light-Harvesting Complex II on Ion Transport across Model Lipid Membranes. J. Photochem. Photobiol. B Biol. 2000, 56 (1), 12-18.

(17) Chmeliov, J.; Gelzinis, A.; Songaila, E.; Augulis, R.; Duffy, C. D. P.; Ruban, A. V.; Valkunas, L. The Nature of Self-Regulation in Photosynthetic Light-Harvesting Antenna. Nat. Plants 2016, 2 (5), 16045.

(18) Schlau-Cohen, G. S.; Wang, Q.; Southall, J.; Cogdell, R. J.; Moerner, W. E. Single-Molecule Spectroscopy Reveals Photosynthetic LH2 Complexes Switch between Emissive States. Proc. Natl. Acad. Sci. 2013, 110 (27), 10899-10903.

(19) Krüger, T. P. J.; Ilioaia, C.; van Grondelle, R. Fluorescence Intermittency from the Main Plant Light-Harvesting Complex: Resolving Shifts between Intensity Levels. J. Phys. Chem. B 2011, 115 (18), 5071-5082.

(20) Gruber, M. J.; Chmeliov, J.; Krüger, T. P. J.; Valkunas, L.; van Grondelle, R. Singlet-Triplet Annihilation in Single LHCII Complexes. Phys. Chem. Chem. Phys. 2015, 17 (30), 1984419853.

(21) Kondo, T.; Pinnola, A.; Chen, W. J.; Dall'Osto, L.; Bassi, R.; Schlau-Cohen, G. S. SingleMolecule Spectroscopy of LHCSR1 Protein Dynamics Identifies Two Distinct States Responsible for Multi-Timescale Photosynthetic Photoprotection. Nat. Chem. 2017, 9 (8), 772778.

(22) Tutkus, M.; Chmeliov, J.; Rutkauskas, D.; Ruban, A. V.; Valkunas, L. Influence of the Carotenoid Composition on the Conformational Dynamics of Photosynthetic Light-Harvesting Complexes. J. Phys. Chem. Lett. 2017, 8 (23), 5898-5906.

(23) Rigaud, J.-L.; Lévy, D. Reconstitution of Membrane Proteins into Liposomes. In Methods in Enzymology; Academic Press, 2003; Vol. 372, pp 65-86.

(24) Barros, T.; Kühlbrandt, W. Crystallisation, Structure and Function of Plant Light-Harvesting Complex II. Biochim. Biophys. Acta - Bioenerg. 2009, 1787 (6), 753-772. 
(25) Schaller, S.; Latowski, D.; Jemioła-Rzemińska, M.; Dawood, A.; Wilhelm, C.; Strzałka, K.; Goss, R. Regulation of LHCII Aggregation by Different Thylakoid Membrane Lipids. Biochim. Biophys. Acta - Bioenerg. 2011, 1807 (3), 326-335.

(26) Wilk, L.; Grunwald, M.; Liao, P.-N.; Walla, P. J.; Kuhlbrandt, W. Direct Interaction of the Major Light-Harvesting Complex II and PsbS in Nonphotochemical Quenching. Proc. Natl. Acad. Sci. 2013, 110 (14), 5452-5456.

(27) Niu, S. L.; Doctrow, B.; Mitchell, D. C. Rhodopsin Activity Varies in Proteoliposomes Prepared by Different Techniques. Biochemistry 2009, 48 (1), 156-163.

(28) Larsen, J.; Hatzakis, N. S.; Stamou, D. Observation of Inhomogeneity in the Lipid Composition of Individual Nanoscale Liposomes. J. Am. Chem. Soc 2011, 133, 10685-10687.

(29) Walsh, S. M.; Mathiasen, S.; Christensen, S. M.; Fay, J. F.; King, C.; Provasi, D.; Borrero, E.; Rasmussen, S. G. F.; Fung, J. J.; Filizola, M.; et al. Single Proteoliposome High-Content Analysis Reveals Differences in the Homo-Oligomerization of GPCRs. Biophys. J. 2018, 115 (2), 300-312.

(30) Moya, I.; Silvestri, M.; Vallon, O.; Cinque, G.; Bassi, R. Time-Resolved Fluorescence Analysis of the Photosystem II Antenna Proteins in Detergent Micelles and Liposomes. Biochemistry 2001, 40 (42), 12552-12561.

(31) Natali, A.; Gruber, J. M.; Dietzel, L.; Stuart, M. C. A.; Van Grondelle, R.; Croce, R. LightHarvesting Complexes (LHCs) Cluster Spontaneously in Membrane Environment Leading to Shortening of Their Excited State Lifetimes. J. Biol. Chem. 2016, 291 (32), 16730-16739.

(32) Crisafi, E.; Pandit, A. Disentangling Protein and Lipid Interactions That Control a Molecular Switch in Photosynthetic Light Harvesting. Biochim. Biophys. Acta-Biomembr. 2017, 1859 (1), $40-47$.

(33) Petrou, K.; Belgio, E.; Ruban, A. V. PH Sensitivity of Chlorophyll Fluorescence Quenching Is Determined by the Detergent/Protein Ratio and the State of LHCII Aggregation. Biochim. Biophys. Acta - Bioenerg. 2014, 1837 (9), 1533-1539.

(34) Mathiasen, S.; Christensen, S. M.; Fung, J. J.; Rasmussen, S. G. F.; Fay, J. F.; Jorgensen, S. K.; Veshaguri, S.; Farrens, D. L.; Kiskowski, M.; Kobilka, B.; et al. Nanoscale High-Content Analysis Using Compositional Heterogeneities of Single Proteoliposomes. Nat. Methods 2014, $11(9), 931-934$.

(35) Cowell, G. M.; Tranum-Jensen, J.; Sjöström, H.; Norén, O. Topology and Quaternary Structure of Pro-Sucrase/Isomaltase and Final-Form Sucrase/Isomaltase. Biochem. J. 1986, 237 (2), 455461.

(36) Tsekouras, K.; Custer, T. C.; Jashnsaz, H.; Walter, N. G.; Pressé, S. A Novel Method to Accurately Locate and Count Large Numbers of Steps by Photobleaching. Mol. Biol. Cell 2016, 27 (22), 3601-3615. 
(37) Kunding, A. H.; Mortensen, M. W.; Christensen, S. M.; Stamou, D. A Fluorescence-Based Technique to Construct Size Distributions from Single-Object Measurements: Application to the Extrusion of Lipid Vesicles. Biophys. J. 2008, 95 (3), 1176-1188.

(38) Gullapalli, R. R.; Demirel, M. C.; Butler, P. J. Molecular Dynamics Simulations of DiI-C18(3) in a DPPC Lipid Bilayer. Phys. Chem. Chem. Phys. 2008, 10 (24), 3548.

(39) Diao, J.; Ishitsuka, Y.; Lee, H.; Joo, C.; Su, Z.; Syed, S.; Shin, Y. K.; Yoon, T. Y.; Ha, T. A Single Vesicle-Vesicle Fusion Assay for in Vitro Studies of Snares and Accessory Proteins. Nat. Protoc. 2012, 7 (5), 921-934.

(40) Bendix, P. M.; Pedersen, M. S.; Stamou, D. Quantification of Nano-Scale Intermembrane Contact Areas by Using Fluorescence Resonance Energy Transfer. Proc. Natl. Acad. Sci. 2009, 106 (30), 12341-12346.

(41) Caffarri, S.; Croce, R.; Breton, J.; Bassi, R. The Major Antenna Complex of Photosystem II Has a Xanthophyll Binding Site Not Involved in Light Harvesting. J. Biol. Chem. 2001, 276 (38), 35924-35933.

(42) Berthold, D. A.; Babcock, G. T.; Yocum, C. F. A Highly Resolved, Oxygen-Evolving Photosystem II Preparation from Spinach Thylakoid Membranes. EPR and Electron-Transport Properties. FEBS Lett. 1981, 134 (2), 231-234.

(43) Porra, R. J.; Thompson, W. A.; Kriedemann, P. E. Determination of Accurate Extinction Coefficients and Simultaneous Equations for Assaying Chlorophylls a and b Extracted with Four Different Solvents: Verification of the Concentration of Chlorophyll Standards by Atomic Absorption Spectroscopy. BBA - Bioenerg. 1989, 975 (3), 384-394.

(44) Lambert, O.; Levy, D.; Ranck, J. L.; Leblanc, G.; Rigaud, J. L. A New “gel-like” Phase in Dodecyl Maltoside-Lipid Mixtures: Implications in Solubilization and Reconstitution Studies. Biophys. J. 1998.

(45) Tutkus, M.; Marciulionis, T.; Sasnauskas, G.; Rutkauskas, D. DNA-Endonuclease Complex Dynamics by Simultaneous FRET and Fluorophore Intensity in Evanescent Field. Biophys. J. 2017, 112 (5), 850-858.

(46) Tutkus, M.; Chmeliov, J.; Rutkauskas, D.; Ruban, A. V.; Valkunas, L. Influence of the Carotenoid Composition on the Conformational Dynamics of Photosynthetic Light-Harvesting Complexes. J. Phys. Chem. Lett. 2017, 8 (23), 5898-5906.

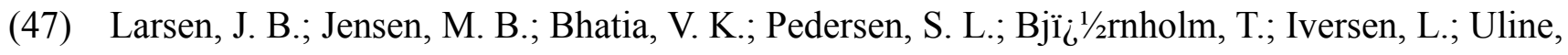
M.; Szleifer, I.; Jensen, K. J.; Hatzakis, N. S.; et al. Membrane Curvature Enables N-Ras Lipid Anchor Sorting to Liquid-Ordered Membrane Phases. Nat. Chem. Biol. 2015, 11 (3), 192-194.

(48) Axelrod, D. Evanescent Excitation and Emission in Fluorescence Microscopy. Biophys. J. 2013, 104 (7), 1401-1409. 
(49) Ruban, A. V.; Horton, P. Mechanism of $\Delta \mathrm{pH}-$ Dependent Dissipation of Absorbed Excitation Energy by Photosynthetic Membranes. I. Spectroscopic Analysis of Isolated Light-Harvesting Complexes. BBA - Bioenerg. 1992, 1102 (1), 30-38.

(50) Rigaud, J. L.; Paternostre, M. T.; Bluzat, A. Mechanisms of Membrane Protein Insertion into Liposomes during Reconstitution Procedures Involving the Use of Detergents. 2. Incorporation of the Light-Driven Proton Pump Bacteriorhodopsin. Biochemistry 1988, 27 (8), 2677-2688.

(51) Hatzakis, N. S.; Bhatia, V. K.; Larsen, J.; Madsen, K. L.; Bolinger, P.-Y.; Kunding, A. H.; Castillo, J.; Gether, U.; Hedegård, P.; Stamou, D. How Curved Membranes Recruit Amphipathic Helices and Protein Anchoring Motifs. Nat. Chem. Biol. 2009, 5 (11), 835-841.

(52) Stamou, D.; Duschl, C.; Delamarche, E.; Vogel, H. Self-Assembled Microarrays of Attoliter Molecular Vessels. Angew. Chemie - Int. Ed. 2003, 42 (45), 5580-5583.

(53) Boukobza, E.; Sonnenfeld, A.; Haran, G. Immobilization in Surface-Tethered Lipid Vesicles as a New Tool for Single Biomolecule Spectroscopy. J. Phys. Chem. B 2001.

(54) Lasic, D. D. A Molecular Model for Vesicle Formation. Biochim. Biophys. Acta 1982, 692 (3), 501-502.

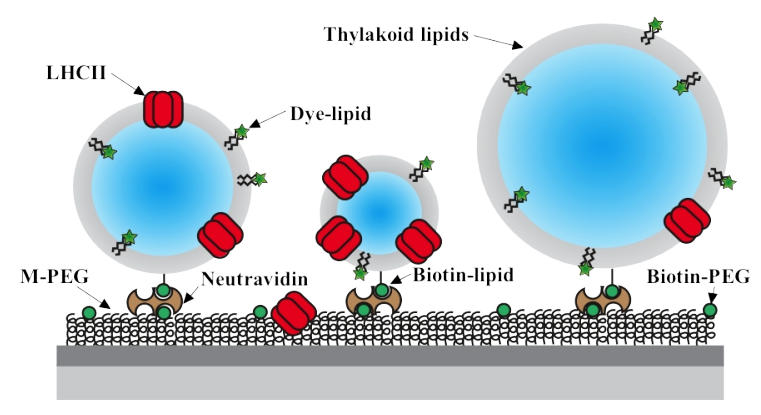



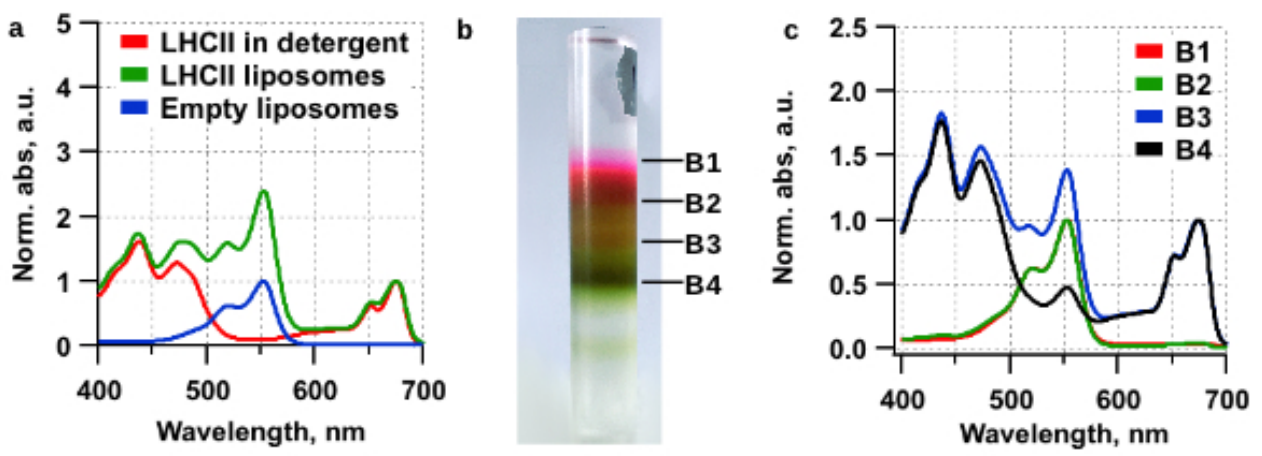

Figure 1 | LHCII proteoliposome fractions and their absorption spectra. a, Absorption spectra of control liposomes, LHCII trimers in detergent micelles, and LHCII proteoliposomes at L:P ratio of the reconstitution mixture being 500:1. The latter two spectra are normalized at $674 \mathrm{~nm}$. b, Separation of LHCII liposome fractions by gradient density. Differently colored bands of increasing density in ficoll are denoted as B1-B4. c, Absorption spectra of proteoliposome sample bands B1-B4 from reconstitution mixture with L:P ratio of 1500:1. The spectra of B3-B4 bands are normalized at $674 \mathrm{~nm}$. 
a
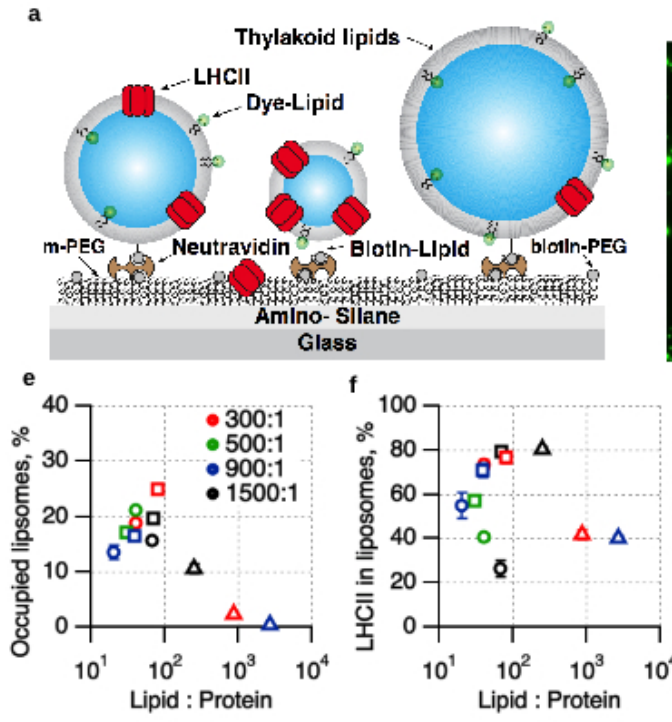
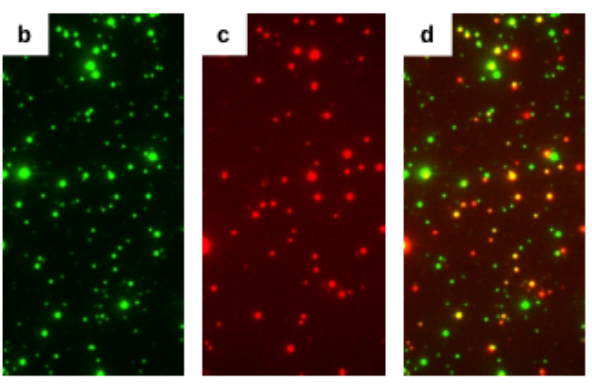

g

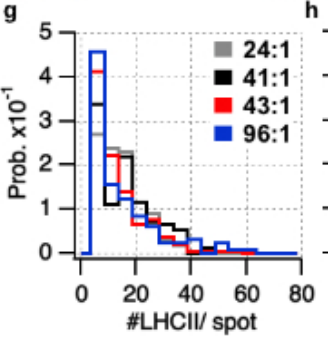

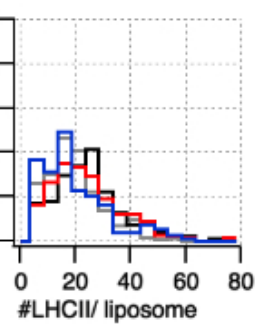

Figure 2 | TIRF microscopy of single LHCII proteoliposomes. a, The experimental scheme showing immobilization of the variously-sized proteoliposomes that contain both DiI dyes and biotin-lipids on the silanized and PEGylated (10\% biotin-PEG) glass coverslip via Neutravidin. b-d, TIRF images obtained via the DiI channel at 532nm excitation (b), LHCII channel at $635 \mathrm{~nm}$ (c) and both channels overlaid (d).

e, Percentage of the occupied liposomes vs. lipid/protein ratio of the gradient band. Colors indicate L: $\mathrm{P}(\mathrm{rm})$ of the reconstitution mixture, symbols-different gradient bands: B2 (triangles), B3 (squares) and B4 (circles). f, Percentage of reconstituted LHCII complexes vs. L:P(gb) of the gradient band. Color in symbol notation is the same as for panel $\mathbf{e}$. $\mathbf{g}-\mathbf{h}$, The statistical distribution of the number of LHCII complexes per spot representing the free surface-bound LHCIIs $\mathbf{( g )}$ and per liposome (h) in the TIRF image, calculated from bleaching traces for four distinct $L: P(g b)$ ratios shown in the legend. 

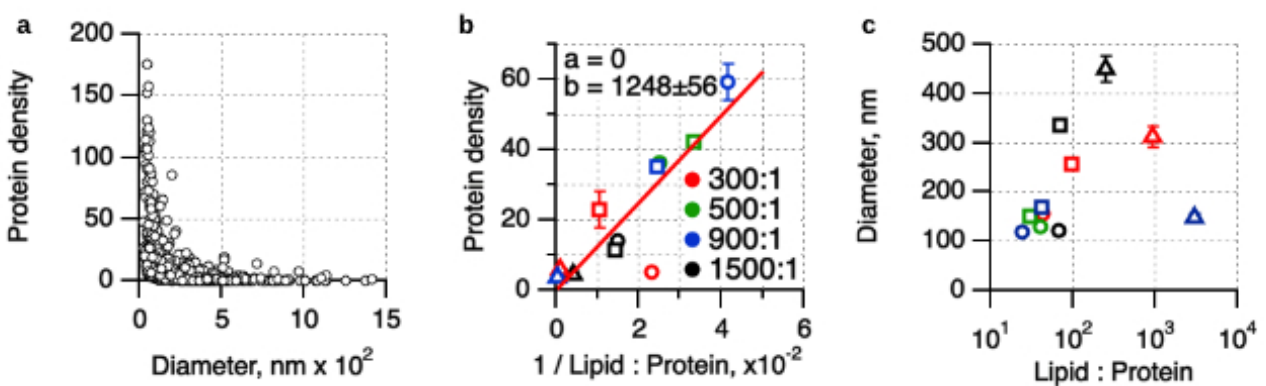

Figure 3 | Confocal microscopy of single LHCII proteoliposomes. a, Relation between the protein density in the proteoliposome (the ratio of fluorescence intensity of LHCII and liposome dyes) and the liposome diameter. $\mathbf{b}$, Linear correlation between the mean protein density in the proteoliposome and the inverted $L: P(g b)$ ratio of the gradient band. $\mathbf{c}$, Average liposome diameter vs. $L: P$ of the gradient band. 

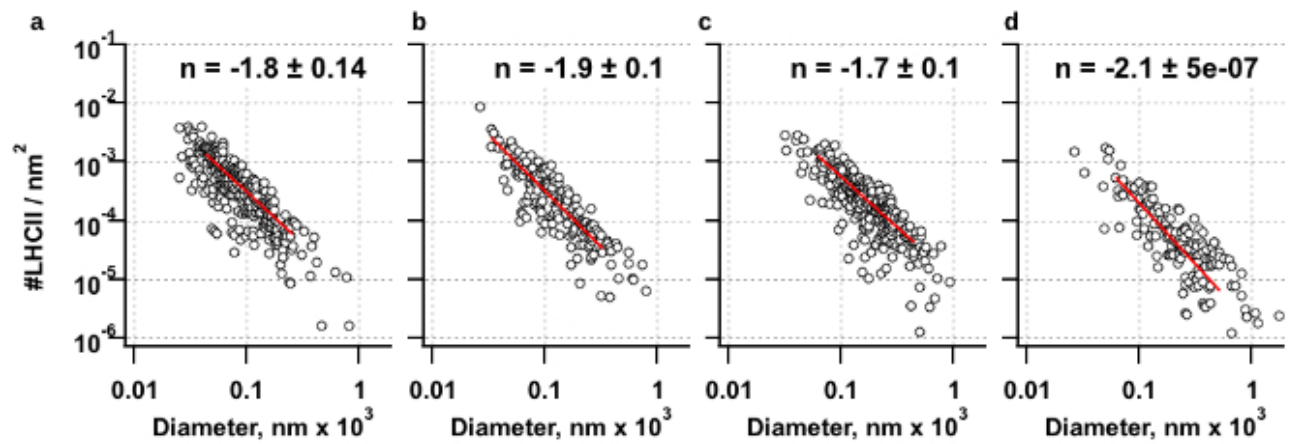

Figure 4 | Distribution of the LHCII mean surface concentration (the number of LHCII trimers per unit surface area of the proteoliposome) for variously sized liposomes. The panels correspond to different lipid/protein ratios of the B3 and B4 gradient bands from different reconstitution mixtures: L:P(gb) $=24: 1$ (a) , 41:1 (b), 43:1 (c), and 96:1 (d). Red lines demonstrate the power-law description, $y \propto x^{n}$, and the exponent $\mathrm{n}$ is indicated in each case. 


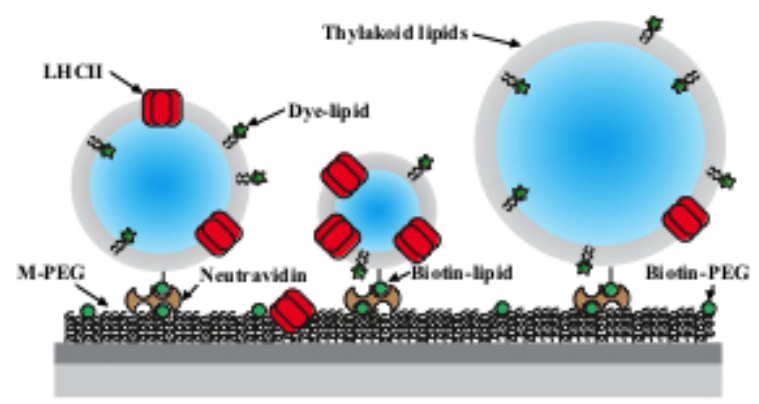

For Table of Contents Only 